\title{
Tratamiento del cáncer de canal anal. Descripción de técnica y resultados de tratamiento radiante con 0 sin quimioterapia. Estudio retrospectivo en una población no seleccionada (1988-2016)
}

José Honorio Leborgne * , Bettys Ortega†, Sergio Aguiarł, María Luisa Terradas*, Valentina Lestido§, Mauro Giordano", Julieta Mezzera**

\section{Resumen}

Introducción: el cáncer de canal anal representa aproximadamente un 2\% a 3\% de todas las neoplasias digestivas. Nigro describió el tratamiento conservador del cáncer de canal anal utilizando radioterapia y quimioterapia con 5-fluorouracilo (5-FU) y mitomicina C (MMC), desde entonces este tratamiento es estándar.

Objetivo: describir la técnica utilizada en nuestro servicio y los resultados en el control locorregional del cáncer de canal anal.

Método: descriptivo, longitudinal, retrospectivo. Se analizaron historias de 72 pacientes consecutivos sin exclusiones, portadores de cáncer de canal anal, entre 1988 y 2016. El 67\% recibió radioquimioterapia y el 33\% de los pacientes radioterapia exclusiva por presentar contraindicaciones médicas específicas y fueron analizados en su conjunto. No se planificaron interrupciones en los tratamientos. Las interrupciones obligatorias fueron de 1 a 17 días con una media de 3 días. Las reacciones agudas fueron moderadas y no se comprobaron complicaciones tardías. Todos recibieron radioterapia locorregional.

Resultados: el control local en N0 fue de $86 \%$ en T1 y T2 versus $42 \%$ en T3 y T4 a seis años, $p=0,01$ significativo.

Conclusiones: la radioterapia con la técnica utilizada en nuestro servicio es efectiva en el tratamiento del cáncer de canal anal. La recidiva local ha sido la causa principal de persistencia lesional o recidiva. La curabilidad de la enfermedad está en relación directa con la extensión clínica/imagenológica de la lesión local.

Palabras clave: Canal anal

Neoplasias del ano

Neoplasias del sistema digestivo

Neoplasias gastrointestinales

Dosificación radioterapéutica
Key words: $\quad$ Anal canal

Anus neoplasms

Digestive system neoplasms

Gastrointestinal neoplasms

Radiotherapy dosage

\footnotetext{
* Oncólogo radioterapeuta.

† Doctor en Medicina.

‡ Oncólogo radioterapeuta y Prof. Agdo. Oncología Radioterápica, Facultad de Medicina, Universidad de la República.

$\S$ Ex Prof. Adj. Licenciada en Enfermería especializada en Radioterapia.

Tl Licenciado en Física médica y Asistente Gr. 2 de Física aplicada. Escuela de Tecnología Médica, Facultad de Medicina, Universidad de la Re-

pública.

** Especialista en Bioestadística.

Correspondencia: Dr. José Honorio Leborgne. Canelones 1165 CP 11100. Montevideo, Uruguay. Correo electrónico: clinicaleborgne@netga-

te.com.uy

Los autores declaran no tener conflicto de intereses.

Recibido: 25/3/19

Aprobado: 5/8/19
} 


\section{Introducción}

El cáncer de canal anal representa aproximadamente un $2 \%$ a $3 \%$ de todas las neoplasias digestivas ${ }^{(1)}$. La incidencia en Uruguay para el período 2007-2011 correspondió a 88 casos, lo que equivale a $0,67 \%$ de todas las neoplasias digestivas en Uruguay, que serían los incluidos en las categorías C21.1 y C21.2 de la Clasificación Internacional de Enfermedades (CIE-10) ${ }^{(2)}$. Los riesgos asociados a una mayor incidencia son: infección por virus del papiloma humano (VPH), infección por virus de la inmunodeficiencia humana (VIH), aumento del número de compañeros sexuales, sexo anorreceptivo, historia de enfermedades transmisión sexual ${ }^{(3)}$.

El canal anal comprende los últimos 3 a $5 \mathrm{~cm}$ del tracto gastrointestinal, a través del esfínter externo e interno, extendiéndose desde la línea pectínea o dentada hasta el reborde anal. El canal anal quirúrgico se extiende desde el margen anal (ano) hasta la línea anorrectal, con una extensión de 4 a $5 \mathrm{~cm}$. El canal anal propiamente dicho, de 2,5 a $3 \mathrm{~cm}$ de largo cráneo-caudal, comprende desde las columnas de Morgagni hasta el margen del ano $^{(4)}$. El ano no se incluye en la clasificación de tumores digestivos por estar recubierto de piel ${ }^{(5)}$. Datos aportados por el Registro Nacional del Cáncer del período 2010-2014 revelan que la distribución por histología del cáncer de canal anal en Uruguay es de 72,5\% carcinomas epidermoides (incluidos los basaloides y cloacogénicos), $18,7 \%$ adenocarcinomas, $5,3 \%$ de casos sin registro de histología, $3,2 \%$ de carcinomas sin especificar. Respecto a las histologías infrecuentes (melanomas, sarcomas, carcinomas de células pequeñas, linfomas, etcétera) solamente se registró un caso de tumor carcinoide en ese período ${ }^{(6)}$.

Previo al tratamiento conservador con radioterapia y quimioterapia, el tratamiento de elección era la cirugía radical. Nigro y colaboradores, en su trabajo pionero, consolidaron la posibilidad del tratamiento conservador del cáncer de canal anal utilizando radioterapia y quimioterapia con 5 -fluorouracilo (5-FU) y mitomicina $\mathrm{C}$ $(\mathrm{MMC})^{(7)}$, desde entonces este tratamiento es el recomendado. Con radioterapia externa exclusiva la dosis recomendada al tumor primario es de 60 a 65 Gy en 6 a 7 semanas ${ }^{(8)}$. Cuando la irradiación se administra en forma concurrente con la quimioterapia, la mitad de los pacientes tratados requieren interrupción del tratamiento por toxicidad aguda de enteroproctitis o dermatitis perineal $^{(8)}$.

A nivel nacional, investigaciones anteriores son las de Torres y colaboradores ${ }^{(9)}$, quienes analizaron retrospectivamente los resultados de la radioquimioterapia en esta patología en 20 pacientes tratados entre los años 1989 y 1994, con $80 \%$ de remisión completa. Torres ${ }^{(10)}$ también analizó resultados del tratamiento radioquimioterápico en 50 pacientes tratados entre enero de 1989 y diciembre de 2000, donde registró una sobrevida global de $80 \%$ a los cinco años.

La utilización terapéutica de las radiaciones en el tratamiento del cáncer se basa en utilizar la acción diferencial de la misma sobre el tejido sano y el tumoral. Para que el tejido sano se recupere y las células reparen las lesiones subletales es imprescindible la existencia de un intervalo de tiempo entre fracciones, permitiendo una regeneración de las células tumorales, lo que puede suponer una limitación para el control tumoral por radioterapia. Aproximadamente un tercio de la radiación administrada en cada fracción se emplea en destruir el porcentaje de células tumorales que se han regenerado desde la fracción precedente ${ }^{(11)}$.

Para optimizar un tratamiento de radioterapia podemos modificar tres parámetros: la dosis total, la dosis por fracción y el intervalo de tiempo entre fracciones. Esto evidencia la necesidad de definir una magnitud que contemple estas variaciones a la hora de lograr el mismo efecto biológico sobre un tejido. A dicha magnitud se la denomina dosis biológica efectiva (BED) y está basada en el modelo lineal-cuadrático ${ }^{(12)}$ :

$$
B E D_{10}=n \cdot d \cdot\left(1+\frac{d}{\alpha / \beta}\right)-\frac{\operatorname{Ln}(2)}{\alpha} \cdot \frac{\left(T-T_{k}\right)}{T_{p}}
$$

Donde $n$ es el número de fracciones, $d$ es la dosis por fracción (Gy), $T_{k}$ es el momento (días desde el comienzo del tratamiento) en que comienza la repoblación acelerada, $T$ son los días totales de tratamiento ${ }^{-1}, T_{p}$ es el tiempo potencial de repoblación celular.

\section{Se asume que:}

$$
\frac{\alpha}{\beta}=10 G y, \alpha=0,3 G y^{-1}, \text { y } T_{k}=28 \text { días y } T_{p}=3 \text { días. }
$$

La técnica más comúnmente utilizada para irradiar el canal anal consta de la irradiación de toda la pelvis mediante extensos campos anteroposteriores. El borde superior del campo se localiza en la unión lumbosacra. El borde inferior se ubica a $3 \mathrm{~cm}$ por debajo del tumor. Los bordes laterales dependen del volumen a tratar y se prefiere minimizar la irradiación en la cabeza y cuello femoral. Otra técnica que sugiere la bibliografía es la irradiación de la pelvis a través de un campo posterior al tumor primario con irradiación de ganglios inguinales con campos de volumen reducido en comparación con la técnica anterior. Los ganglios inguinales son a menudo tratados con electrones ${ }^{(8)}$.

El canal anal puede ser tratado por tres o cuatro campos análogos a los utilizados en tratamiento de cáncer de recto $^{(13)}$. La alta toxicidad de utilizar campos pelvianos 
que incluyen genitales y campos de sobreimpresión (boost) sobre el tumor y los ganglios, ha llevado a la recomendación de utilizar la radioterapia de intensidad modulada (IMRT) de acuerdo al estudio RTOG 0529 (fase II $)^{(14)}$. Esta ha disminuido las secuelas en vejiga, recto, intestino delgado, genitales, cabezas femorales y la piel perineal. El desarrollo de la IMRT representa una técnica más precisa en cuanto a la 3D con la posibilidad de lograr una mejor protección de estructuras sanas, y, por lo tanto, una mejor tolerancia y así evitar o disminuir las interrupciones programadas o necesarias. El panel de consenso de RTOG ha diseñado un atlas sobre la base de un cáncer de recto T3N2 a $7 \mathrm{~cm}$ del margen anal ${ }^{(15)}$, los autores de este atlas advierten las dificultades que presenta para el radioncólogo el diseño de contornos.

El objetivo general es describir la técnica utilizada en nuestro servicio y los resultados en el control locorregional del cáncer de canal anal.

Como objetivos específicos planteamos:

1. Evaluar la incidencia de recidiva locorregional.

2. Cuantificar los efectos de las interrupciones programadas.

3. Implementar el modelo lineal cuadrático para calcular la dosis biológica efectiva (DBE) en nuestra población.

4. Evaluar la presencia de toxicidad aguda en tejidos sanos.

5. Evaluar la presencia de complicaciones tardías.

\section{Material y método}

Estudio descriptivo, longitudinal, retrospectivo. Se revisaron las historias clínicas de pacientes con cáncer de canal anal tratados con radioterapia entre febrero de 1988 y junio de 2016.

Se incluyeron todos los pacientes vistos y tratados con radioterapia. Se excluyeron los pacientes con cáncer de ano y pacientes con cáncer de recto.

No se ha hecho ninguna selección ni exclusión de pacientes entre aquellos que eran aptos para el tratamiento de radioquimioterapia o aquellos que no lo eran debido a sus comorbilidades; por lo tanto, obtendremos una visión más completa de la realidad de la enfermedad.

Se trataron 72 pacientes, de los cuales 54 eran mujeres $(75 \%)$ y 18 eran hombres (25\%) en un rango de edad de 41 a 92 años (mediana de 67 años). La anatomía patológica correspondió a 64 casos de carcinoma epidermoide (89\%), 7 casos de adenocarcinoma (10\%), 1 caso de carcinoma indiferenciado (1\%). La distribución de pacientes según los estadios fue de 10 pacientes con estadio I, 35 con estadio II, 6 con estadio IIIA y 21 con estadio IIIB.
Todos los pacientes tuvieron confirmación histopatológica y fueron reestadificados según la clasificación del American Joint Committee on Cancer (AJCC, séptima edición $)^{(9)}$.

En los últimos años hubo una evolución en los tratamientos radioterápicos que llevaron a una mayor precisión, disminuyendo su toxicidad.

Hemos tratado el cáncer de canal anal al inicio con unidades de cobaltoterapia $\left(\mathrm{Co}^{60}\right)$ isocéntrico a $80 \mathrm{~cm}$ hasta el año 1989 (tres pacientes) con cálculos manuales y dosimetría 2D y campos anteroposteriores y laterales en decúbito ventral. Desde el año 1989, se utilizó planificación con tomografía computarizada (TC) en decúbito ventral, con marcador en regiones inguinales y en canal anal, además de medio de contraste intrarrectal, dosimetría 3D y fotones de $6 \mathrm{MV}$ (12 pacientes). A partir del año 2002, se utilizaron fotones de 6 o 15 MV y dosimería 3D conformal y planificación con equipo XIO 4.44.02, así como electrones en campos adicionales en adenopatías inguinales y a partir del año 2016 se utilizó el sistema de contorneo y fusión de imágenes Monaco Sim 5.11.02 y el sistema de planificación XIO 5.10.02.

En el curso de estos años fueron utilizados cuatro equipos de TC con los progresos imagenológicos correspondientes. Desde el año 1989, a todos los pacientes se les realizó TC de abdomen y pelvis, y en ninguno de ellos se observaron adenopatías pelvianas, por lo tanto todos los tratamientos fueron locorregionales. Se utilizaron tres campos de irradiación (uno posterior y dos oblicuos anteriores) hasta una dosis blanco de 60 Gy para un volumen de 350 a $400 \mathrm{ml}$ y de 25 a 28 Gy en las regiones inguinales (en los pacientes N0). Para los pacientes con $\mathrm{N} 2$ o N3 se utilizaron campos directos inguinales adicionales y concomitantes con electrones hasta completar $60 \mathrm{~Gy}$, evitando en todos los casos los genitales en ambos sexos y las articulaciones coxofemorales.

La dosis en el tumor tuvo una mediana de $60 \mathrm{~Gy}$ (con un rango de 50 a 72 Gy) en una mediana de tiempo de 46 días (con un rango de 36 a 84 días). Solo cuatro pacientes recibieron dosis altas de entre 70 y 72 Gy por los siguientes motivos: persistencia lesional en dos casos, uno que al completar los 60 Gy recibió un complemento local de 10 Gy por no mostrar respuesta y el otro era un paciente de 81 años y lesión T4N2 que no recibió quimioterapia.

Los casos fueron referidos por más de 20 oncólogos médicos y de diferentes instituciones de salud del país. Los planes de tratamiento fueron (5FU exclusivo con distintos protocolos, $\mathrm{MMC}$ y $5 \mathrm{FU}, 5 \mathrm{FU}$ y cisplatino). $\mathrm{La}$ quimioterapia dependió de la decisión de los oncólogos que enviaron a los pacientes, por lo que no permite subdividir en subgrupos según la quimioterapia que recibieron, pero sí subdividir entre los que recibieron quimiote- 


\begin{tabular}{|lcc|}
\hline \multicolumn{3}{|l|}{ Tabla 1. Regresión de Cox. Control local. } \\
\hline Variable & $95 \%$ IC & $p$ \\
\hline QT & $0,6521-3,6354$ & 0,325 \\
Dosis & $0,9992-1,0004$ & 0,604 \\
Estadio & $0,7226-2,2055$ & 0,4130 \\
\hline
\end{tabular}

Tabla 3. Distribución de pacientes según T.

\begin{tabular}{lcc}
\hline Sexo & $F A$ & $F R(\%)$ \\
\hline Mujeres & 54 & 75 \\
Hombres & 18 & 25 \\
Total & 72 & 100 \\
\hline
\end{tabular}

Tabla 5. Distribución de pacientes según estadio.

\begin{tabular}{lll|}
\hline Estadio & $F A$ & $F R(\%)$ \\
\hline I & 10 & 13,9 \\
II & 35 & 48,6 \\
III-A & 6 & 8,3 \\
III-B & 21 & 29,2 \\
Total & 72 & 100 \\
\hline
\end{tabular}

rapia (67\%) y los que no recibieron quimioterapia (33\%) por contraindicaciones médicas específicas analizadas en su conjunto.

No se planificó ninguna interrupción en los tratamientos, aunque las intolerancias a ambas modalidades terapéuticas obligaron en algunos casos a suspenderlos con interrupciones que modificaron la BED. Dichas interrupciones tienen una mediana de 3 días con un mínimo de 1 día y un máximo de 17 días.

\section{Consideraciones éticas}

Este estudio se realizó siguiendo las pautas éticas de la Declaración de Helsinki del año $2000^{(16)}$ y en el marco del Decreto Ley 379/008 para investigaciones con seres humanos ${ }^{(17)}$, así como en la Ley 18.331 (de Protección de Datos Personales y de Acción de Habeas Data) ${ }^{(18)}$.

Este estudio fue aprobado por el Comité de Ética de la Médica Uruguaya.
Tabla 2. Distribución de pacientes según sexo.

\begin{tabular}{lcc}
\hline Sexo & $F A$ & $F R(\%)$ \\
\hline Mujeres & 54 & 75 \\
Hombres & 18 & 25 \\
Total & 72 & 100 \\
\hline
\end{tabular}

Tabla 4. Distribución de pacientes según $\mathrm{N}$.

\begin{tabular}{lc}
\hline$N$ & $F A$ \\
\hline N0 & 51 \\
N1 & 0 \\
N2 & 14 \\
N3 & 7 \\
Total & 72 \\
\hline
\end{tabular}

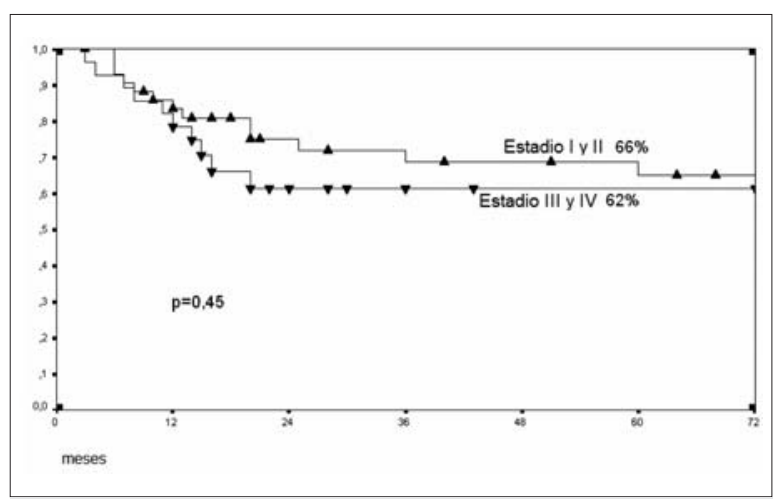

Figura 1. Control local en estadios I y II vs III y IV.

\section{Análisis estadístico}

Se utilizó el programa estadístico SPSS (SPSS Inc, Chicago, USA) para el análisis multivariado del modelo de Cox (tabla 1) y el análisis actuarial de Kaplan-Meier. Con este modelo se obtuvieron gráficas de control local para estadios I y II versus estadios III y IV, control local en N0 para T1 y T2 versus N0 para T3 y T4, sobrevida libre de enfermedad en estadios I y II versus III y IV, control ganglionar para estadios I y II versus III y IV, y el cálculo de la BED10 individual para determinar el control local.

Se realizaron tablas simples para sexo (tabla 2), $\mathrm{T}$ (tabla 3), N (tabla 4) y estadio (tabla 5). 


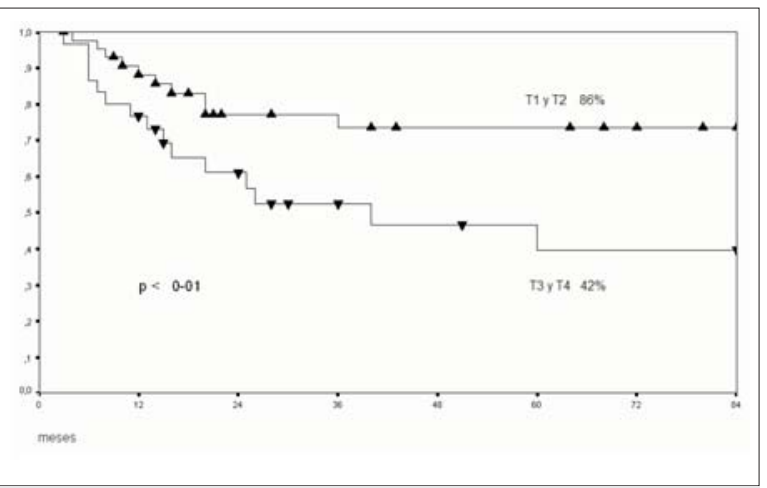

Figura 2. Control local para $n=0$ en $\mathrm{T} 1$ y T2 vs T3 y T4.

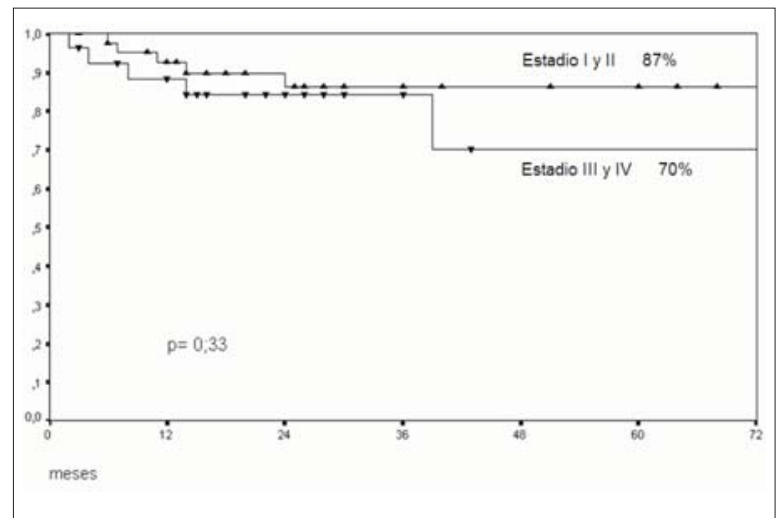

Figura 4. Sobrevida libre de enfermedad en estadios I y II vs III y IV.

\section{Resultados}

Se obtuvo un control local de $66 \%$ para los estadios I y II y para los estadios III y IV, que fue de $62 \%$; $p=0,45$ no significativo (NS) (figura 1).

El control local para los pacientes con N0 fue de $86 \%$ (T1 y T2) y $42 \%$ (T3 y T4); $\mathrm{p}=0,01$ significativo (figura 2 ).

La sobrevida libre de enfermedad a seis años correspondió a $61 \%$ en los estadios I y II y en los estadios III y IV correspondió a 57\%; $\mathrm{p}=0,52 \mathrm{NS}$ (figura 3).

El control ganglionar para los estadios I y II fue de $87 \%$ y para los estadios III y IV fue de $70 \%$; $p=0,33$ no significativo (figura 4).

Los pacientes fallecidos por la enfermedad fueron $21(29,2 \%)$, los fallecidos por causa intercurrente fueron $9(12,5 \%)$ y los fallecidos por un segundo cáncer fueron $3(4,2 \%)$.

En cuanto a las complicaciones agudas (según las escalas de toxicidad aguda del Radiation Therapy Oncology Group - Cooperative Group Common Toxicity Criteria), 16 pacientes presentaron radiodermitis húmeda

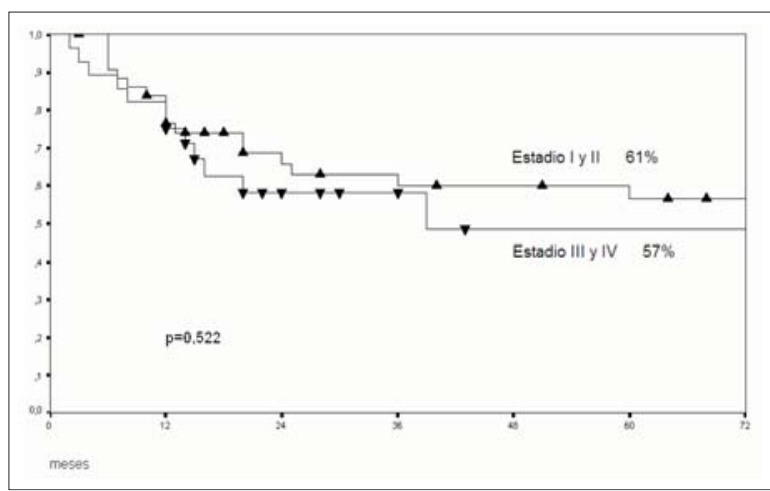

Figura 3. Sobrevida libre de enfermedad en estadios I y II vs III y IV.

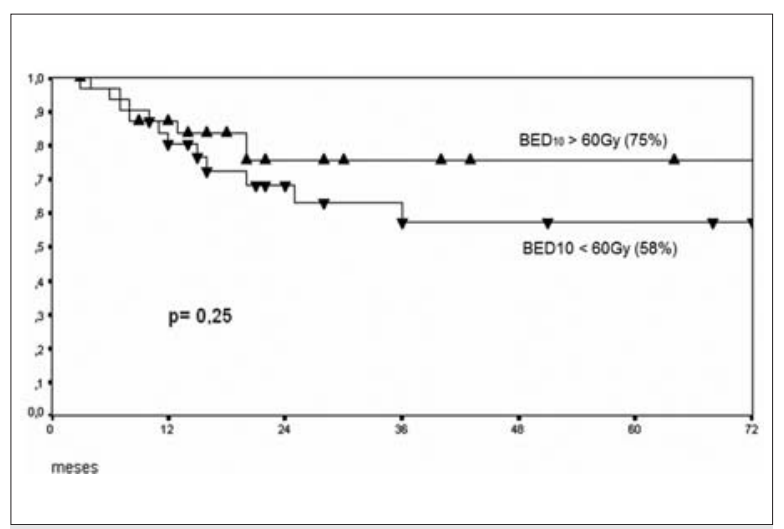

Figura 5. Control local para BED $<60 \mathrm{~Gy}_{10}$ vs BED $\geq$ $60 \mathrm{~Gy}_{10}$.

(grado 3 RTOG), 5 presentaron diarrea (grado 2 RTOG), 3 presentaron diarrea (grado 2 RTOG) y radiodermitis húmeda (grado 3 RTOG) y uno solo tuvo incontinencia fecal (grado 3 RTOG). No se detectaron complicaciones tardías y hubo cinco pacientes perdidos de vista en los primeros cinco años.

Cinco pacientes presentaron metástasis fuera del campo de irradiación: un caso T2N2 tratado en febrero de 2012 y reirradiado en octubre de 2015 por metástasis ganglionar pararrectal derecha y actualmente en remisión completa; un caso T3N0 tratado en julio de 2014 y fallecido por metástasis simultáneas pelvianas y abdominales; un caso T3N0 tratado en agosto de 2015 que presentó metástasis ganglionar presacra reirradiado en enero de 2017; un caso T2N3 tratado en setiembre de 2014 y reirradiado en octubre de 2016 por metástasis ósea en pelvis; un caso T4N2 tratado en enero de 2014 y reirradiado en febrero de 2017 por metástasis ilíaca primitiva izquierda. 
Utilizando los parámetros radiobiológicos obtenidos por Fowler para este propósito ${ }^{(12)}$, hemos calculado una pérdida de 0,66 Gy diarios a partir de la tercera semana de tratamiento en cáncer de canal anal con histología epidermoide.

Por otra parte, en el estudio del BED realizado en todos los carcinomas epidermoides se obtuvo que con una dosis mayor o igual a la mediana de $60 \mathrm{~Gy}_{10}$ se obtiene un control local de $75 \%$ versus $58 \%$ para las dosis inferiores, $\mathrm{p}=0,25$, no significativo (figura 5 ).

\section{Discusión}

La literatura internacional considera la radioterapia y quimioterapia con 5-FU/MMC como el tratamiento recomendado, quedando excluidos de este tratamiento aquellos que tienen contraindicaciones médicas a la quimioterapia, que en nuestro estudio corresponden a $33 \%$ de los casos. En nuestro medio se publicaron dos trabajos $^{(9,10)}$ tratados con unidades de cobaltoterapia y todos con 5-FU/MMC, por lo que sus resultados no son comparables con este estudio.

En los estudios revisados por los autores Glynne-Jones y $\mathrm{Rao}^{(19)}$ se relata que la recaída locorregional es predominante en la recurrencia, comprobada tanto en estudios retrospectivos como en ensayos clínicos randomizados, siendo responsable de la mayor parte de las muertes por la enfermedad ${ }^{(20-23)}$.

Nuestro análisis es concordante con estos estudios, donde la recaída locorregional es la mayor causa de fracasos.

Los ensayos clínicos $\mathrm{ACTI}^{(22)}$ y EORTC ${ }^{(24)}$ demostraron mejoría en el control locorregional así como disminución de la incidencia de colostomías con el plan de radioquimioterapia versus plan de radioterapia exclusiva. A los cinco años, el control locorregional para los grupos de radioquimioterapia versus radioterapia exclusiva fue de $68 \%$ vs $43 \%{ }^{(22)}$ y de $68 \%$ vs $51 \%{ }^{(24)}$, así como cinco años de sobrevida libre de enfermedad con valores de $47 \%$ vs $37 \%{ }^{(22)}$ y de $72 \%$ vs $40 \%{ }^{(24)}$ respectivamente.

El estudio ACT I ${ }^{(22)}$ presenta similar porcentaje de toxicidad aguda y tardía de piel para ambos grupos.

El ensayo clínico UKCCCR ${ }^{(25)}$ compara RT+5- FU/ MMC vs RT exclusiva y muestra una recaída local de $39 \%$ vs $61 \%$ respectivamente, y una sobrevida global a tres años de $65 \%$ vs $58 \%$ no significativa.

Spithoff y colaboradores ${ }^{(26)}$ hacen una revisión de los diferentes estudios randomizados. El estudio UKCCCR ${ }^{(25)}$ 5-FU-MMC+RT vs RT exclusiva: 292 y 285 pacientes; la recaída locorregional a tres años fue de $30 \%$ para el primer grupo vs $53 \%$ para el grupo de RT sola, y la sobrevida global a tres años, $65 \%$ vs $60 \%$ respectivamen- te, no es significativo y no hay datos de la sobrevida libre de enfermedad.

El estudio EORCT ${ }^{(24)}$ relata 32\% de recaída local para el grupo de radioquimioterapia y $48 \%$ en el grupo de radioterapia exclusiva, y una sobrevida global de $58 \%$ y $53 \%$, respectivamente, no es significativo.

La mayoría de los estudios señalan que no hay consenso en la dosis óptima y señalan un efecto deletéreo en las interrupciones programadas o necesarias. Los intervalos programados desde el punto de vista radiobiológico disminuyen la dosis total teniendo en cuenta la repoblación celular constante y acelerada ${ }^{(27)}$. Estos autores demuestran, utilizando la fórmula lineal cuadrática, una pérdida por repoblación acelerada de 0,6 Gy por día, por lo que un aumento de la dosis podría ser posible y razonable utilizando la IMRT sin interrupciones.

En nuestro caso no se realizaron interrupciones programadas para el grupo de pacientes tratados y se calculó una pérdida de 0,66 Gy diarios a partir de la cuarta semana de tratamiento en cáncer de canal anal con histología epidermoide.

La alta toxicidad de utilizar campos pelvianos que incluyen genitales y campos de sobreimpresión (boost) sobre el tumor y los ganglios han llevado a la recomendación de utilizar la radioterapia de intensidad modulada de acuerdo al estudio RTOG $0529^{(28)}$. Esta ha disminuido las secuelas en vejiga, recto, intestino delgado, genitales, cabezas femorales y la piel perianal. El desarrollo de la IMRT representaría una técnica más precisa en cuanto a la 3D, con la posibilidad de lograr una mejor protección de estructuras sanas, y por lo tanto, una mejor tolerancia y así evitar o disminuir las interrupciones programadas o necesarias. El panel de consenso de RTOG ha diseñado un atlas sobre la base de un cáncer de recto T3N2 a $7 \mathrm{~cm}$ del margen anal ${ }^{(15)}$, los autores de este atlas advierten de las dificultades que presenta para el radiooncólogo el diseño de contornos.

El único ensayo clínico prospectivo (fase II) que compara ambas modalidades ${ }^{(14)}$ no logra reducir las reacciones agudas genitourinarias y gastrointestinales (mayores a grado 2) en comparación con el grupo RT/5-FU/MMC del estudio RTOG 98-11 ${ }^{(29)}$, que utilizó radioterapia convencional.

El consenso del panel de expertos recomienda la IMRT sobre la 3D conformal para el tratamiento de cáncer de canal anal ${ }^{(15)}$.

En nuestra experiencia, la técnica utilizada excluye de los campos de tratamiento a los genitales y las articulaciones coxofemorales, obteniendo de esta forma valores bajos de toxicidad. 


\section{Conclusiones}

La radioterapia y la radioquimioterapia son los únicos tratamientos conservadores y efectivos para el tratamiento del cáncer de canal anal en una población no seleccionada de 72 pacientes consecutivos. Con la técnica radiante utilizada en este servicio, la recidiva local ha sido la causa principal de persistencia lesional o recidiva. La curabilidad de la enfermedad está en relación directa con la extensión clínica/imagenológica de la lesión local.

Se obtuvo un control local de $66 \%$ en los estadios I y II y $62 \%$ en los estadios III y IV; $\mathrm{p}=0,45$ (NS) (figura 1 ).

El control local en los pacientes con N0 fue de $86 \%$ (T1 y T2) y $42 \%$ (T3 y T4); $\mathrm{p}=0,01$ significativo (figura 2).

La sobrevida libre de enfermedad a seis años correspondió a $61 \%$ en los estadios I y II y $57 \%$ en los estadios III y IV; $p=0,52$ (NS) (figura 3 ).

El control de regiones ganglionares inguinales en estadios I y II fue de $87 \%$ y $70 \%$ para los estadios III y IV; $\mathrm{p}=0,33$ no significativo (figura 4 ).

Los pacientes fallecidos por la enfermedad fueron $21(29,2 \%)$, los fallecidos por causa intercurrente fueron $9(12,5 \%)$ y los fallecidos por un segundo cáncer fueron $3(4,2 \%)$.

La incidencia de complicaciones agudas es muy baja y sin complicaciones tardías; la sobrevida libre de enfermedad a seis años es comparable con otras series internacionales.

De este estudio surge que la curabilidad de la enfermedad está en relación directa con la extensión clínica/imagenológica de la lesión local.

La BED menor o igual a $60 \mathrm{~Gy}_{10}$, comparada con las mayores a $60 \mathrm{~Gy}_{10}$, favorecería las dosis más altas, aunque las diferencias no son significativas.

\section{Abstract}

Introduction: cancer of the anal canal accounts for approximately 2 to $3 \%$ of all digestive neoplasms. Nigro described the conservative treatment of cancer of the anal canal using radiation and chemotherapy of 5-fluorouracil (5-FU) and mitomycin-C (MMC). Since then, this is the standard therapy.

Objective: to describe the technique used in our service and its results in the locoregional follow up of cancer of the anal canal.

Method: descriptive, longitudinal, retrospective. The medical history of 72 consecutive patients with no exclusions, who were carriers of cancer of the anal canal were analysed between 1988 and 2016. 67\% underwent radiation and chemotherapy and $33 \%$ only radiation therapy due to specific medical contraindications. All cases were comprehensively studied and no interruption of treatments were planned. Mandatory interruptions were from 1 to 17 days long and average length was 3 days. Severe reactions were moderate and no late complications were seen. All patients underwent locoregional radiation therapy.

Results: local control in N0 was $86 \%$ in T1 and T2 versus $42 \%$ in $\mathrm{T} 3$ and $\mathrm{T} 4$ after 6 years $\mathrm{p}=0,01$ meaningful.

Conclusions: radiation therapy with the technique used in our service is effective to treat cancer of the anal canal. Local relapse has been the main cause of lesions or relapse. Curability of the disease is directly linked to the clinical/imaging extent of the local lesion.

\section{Resumo}

Introdução: o câncer de canal anal representa aproximadamente 2 a $3 \%$ das neoplasias digestivas. Nigro descreveu o tratamento conservador do câncer de canal anal utilizando radioterapia e quimioterapia com 5-Fluorouracilo (5-FU) e Mitomicina C (MMC) que tem sido o tratamento padrão.

Objetivo: descrever a técnica utilizada no nosso serviço e os resultados do controle locorregional do câncer de canal anal.

Método: descritivo, longitudinal, retrospectivo. Foram analisados os prontuários de 72 pacientes consecutivos sem exclusões, portadores de câncer de canal anal, do período $1988-2016.67 \%$ receberam radio quimioterapia e $33 \%$ radioterapia exclusivamente devido a contraindicações médicas específicas; os resultados foram analisados conjuntamente. Não se planejou interrupções nos tratamentos. As interrupções obrigatórias foram de 1 a 17 dias com uma média de 3 dias. As reações agudas foram moderadas e não se comprovaram complicações tardias. Todos receberam radioterapia locorregional.

Resultados: o controle local em N0 foi de $86 \%$ em $\mathrm{T} 1$ e T2 versus $42 \%$ em T3 e T 4 a 6 anos $\mathrm{p}=0,01$ significativo.

Conclusões: a radioterapia com a técnica utilizada no nosso Serviço é efetiva no tratamento do câncer de canal anal. A recidiva local foi a causa principal de persistência lesional ou recidiva. A curabilidade da enfermidade está diretamente relacionada com a extensão clínica e das imagens da lesão local.

\section{Bibliografía}

1. Siegel RL, Millar KD, Jemal A. Cancer statistics, 2016. CA Cancer J Clin 2016; 66(1):7-30. Disponible en: http://onlinelibrary.wiley.com/doi/10.3322/caac.21332/epdf [Consulta: 14 julio 2017]. 
2. Barrios E. Incidencia del cáncer en Uruguay. Período 2007-2011. Montevideo: CHLCC, 2015.

3. Frisch M, Glimelius B, van den Brule AJ, Wohlfahrt J, Meijer CJ, Walboomers JM, et al. Sexually transmitted infection as a cause of anal cancer. N Engl J Med 1997; 337(19):1350-8. Disponible en: http://www.nejm.org/doi/ full/10.1056/NEJM199711063371904\#t=article [Consulta: 19 julio 2017].

4. Netter FH. Atlas of human anatomy. 6th. Philadelphia, PA: Elsevier, 2014:371p.

5. Webb SP, Lee CS. Epidermoid cancer of the anal canal. Clin Colon Rectal Surg 2011; 24(3):142-8. doi: 10.1055/ s-0031-1285998.

6. CHLCC. Incidencia del cáncer en el Uruguay: 2007-2011 [Incidencia del cáncer del canal anal]. Montevideo, 2017.

7. Nigro ND, Vaitkevicius VK, Considine B Jr. Combined therapy for cancer of the anal canal: a preliminary report. Dis Colon Rectum 1974; 17(3):354-6.

8. Clifford Chao K, Shab AA. Anal canal. En: Clifford Chao K, Pérez C, Brady L, eds. Radiation oncology: management decisions. 3rd ed. Philadelphia, PA: Wolters Kluwer, 2011:455-68.

9. Torres M, Avondet I, Vazquez J, Santini AE. Cáncer del canal anal. Una neoplasia radioquimiocurable. Cir Urug 1997; 67:140-9.

10. Torres M. Tratamiento conservador del carcinoma epidermoide del canal anal con radioquimioterapia concomitante: experiencia 1989-2000. Rev Oncol 2002; 4(7):370-7.

11. Joiner M, van der Kogel A, eds. Basic clinical radiobiology. 4th ed. Londres: Hodder Arnold, 2009.

12. Fowler JF. The linear-quadratic formula and progress in fractionated radiotherapy. Br J Radiol 1989; 62:679-94.
13. Glimelius B, Pahlman L. Radiation therapy of anal epidermoid carcinoma. Int J Radiat Oncol Biol Phys 1987; 13(3):305-12.

14. Kachnic L, Winter K, Myerson RJ, Goodyear MD, Willins J, Esthappan J, et al. RTOG 0529: a phase 2 evaluation of dose-painted intensity modulated radiation therapy in combination with 5-fluorouracil and mitomycin-C for the reduction of acute morbidity in carcinoma of the anal canal. Int $\mathrm{J}$ Radiation Oncol Biol Phys 2013; 86(1):27-33. doi: 10.1016/ j.ijrobp.2012.09.023.

15. Myerson RJ, Garofalo MC, EI Naqa I, Abrams RA, Apte A, Bosch WR, et al. Elective clinical target volumens for conformal therapy in anorectal cancer: a radiation therapy oncology group consensus panel contouring atlas. Int J Radiat Oncol Biol Phys 2009; 74(3):824-30.

16. Asociación Médica Mundial. Declaración de Helsinki. Principios éticos para las investigaciones médicas en seres humanos. Edimburgo: AMM, 2000.

17. Uruguay. Poder Judicial. Decreto de Ley $N^{\circ} 379 / 008$. Investigaciones con seres humanos. Diario Oficial no 27547 (14 agosto 2008):415-A.

18. Uruguay. Poder Judicial. Ley No 18.331. Protección de datos personales y acción de "Habeas Data". Diario Oficial $\mathrm{n}^{\circ}$ 27549 (18 agosto 2008):437-A.

19. Glynne-Jones R, Rao S. Treatment of the primary tumor in anal canal cancers. Sur Oncol Clin N Am 2017; 26(1):73-90. doi: 10.1016/j.soc.2016.07.003.

20. Das P, Bathia S, Eng C, Ajani JA, Skibber JM, Rodriguez-Bigas MA, et al. Predictors and patterns of recurrence after definitive chemoradiation for anal canal cancer. Int J Radiat Oncol Biol Phys 2007; 68(3):794-800.

21. Wright JL, Patil SM, Temple LK, Minsky BD, Saltz LB, Goodman KA. Squamous cell carcinoma of the anal canal: patterns and predictors of failure and implications for inten-

\section{Contribución de autores}

José Honorio Leborgne https://orcid.org/0000-0002-9899-3615. Investigador principal responsable y médico radioterapeuta tratante de los pacientes involucrados en el estudio.

Sergio Aguiar, https://orcid.org/0000-0003-1176-202X. Médico tratante de los pacientes involucrados en el estudio.

María Luisa Terradas, https://orcid.org/0000-0001-8352-152X. Médico tratante de los pacientes involucrados en el estudio.

Bettys Ortega, https://orcid.org/0000-0003-2362-7896. Médico tratante de los pacientes involucrados en el estudio.

Valentina Lestido, https://orcid.org/0000-0002-8687-2234. Licenciada en Enfermería especializada en radioterapia, coautora del estudio, responsable de la revisión de literatura, procesamiento de resultados y edición del manuscrito.

Julieta Mezzera, https://orcid.org/0000-0002-4335-0823. Bioestadística, responsable de los análisis estadísticos y procesamiento de resultados.

Mauro Giordano, https://orcid.org/0000-0002-4960-8397. Responsable del cálculo de la dosis biológica efectiva individual y procesamiento de resultados. 
sity-modulated radiation treatment planning. Int $\mathrm{J}$ Radiat Oncol Biol Phys 2010; 78(4):1064-72.

22. Northover J, Glynne-Jones R, Sebag-Montefiore D, James R, Meadows H, Wan S, et al. Chemoradiation for the treatment of epidermoid anal cancer: 13-year follow-up of the first randomised UKCCCR Anal Cancer Trial (ACT I). Br J Cancer 2010; 102(7):1123-8.

23. Sebag-Montefiore D, James R, Meadows H, Begum R, Cunningham D, Northover $\mathbf{J}$, et al. The pattern and timing of disease recurrence in squamous cancer of the anus: mature results from the NCRI ACT II trail. J Clin Oncol 2012; 30(15 Suppl):4029.

24. Bartelink H, Roelofsen F, Eschwege F, Rougier P, Bosset JF, Gonzalez DG, et al. Concomitant radiotherapy and chemotherapy is superior to radiotherapy alone in the treatment of locally advanced anal cancer: results of a phase III randomized trail of the European Organization for Research and Treatment of Cancer Radiotherapy and Gastrointestinal Cooperative Groups. J Clin Oncol 1997; 15(5):2040-9.

25. UKCCCR Anal Cancer Trial Working Party. Epidermoid anal cancer: results from the UKCCCR randomised trial of radiotherapy alone versus radiotherapy, 5-fluorouracil, and mitomycin. Lancet 1996; 348(9034):1049-54.

26. Spithoff K, Cummings B, Jonker D, Biagi JJ. Chemoradiotherapy for squamous cell cancer of the anal canal: a systematic review. Clin Oncol (R Coll Radiol) 2014; 26(8):473-87. doi: 10.1016/j.clon.2014.03.005.

27. Glynne-Jones R, Sebag-Montefiore D, Adams R, McDonald A, Gollins S, James R, et al. "Mind the gap"- the impact of variations in the duration of the treatment gap and overall treatment time in the first UK Anal Cancer Trial (ACT I). Int J Radiat Oncol Biol Phys 2011; 81(5):1488-94.

28. Herman JM, Thomas CR. RTOG 0529: intensity modultated radiation therapy and anal cancer, a step in the right direction?. Int J Radiat Oncol Biol Phys 2013; 86(1):8-10.

29. Gunderson L, Moughan J, Ajani JA, Pedersen JE, Winter KA, Benson AB 3rd, et al. Anal carcinoma: impact of TN category of disease on survival, disease relapse, and colostomy failure in US Gastrointestinal Intergroup RTOG 98-11 phase 3 trial. Int J Radiat Oncol Biol Phys 2013; 87(4):638-45. 